\title{
Treatment and posttreatment skeletal effects of rapid maxillary expansion studied with low-dose computed tomography in growing subjects
}

\author{
Roberta Lione, ${ }^{\text {a }}$ Fabiana Ballanti, ${ }^{\text {a }}$ Lorenzo Franchi, ${ }^{\text {b }}$ Tiziano Baccetti, ${ }^{\text {b }}$ and Paola Cozza ${ }^{\mathrm{c}}$ \\ Rome and Florence, Italy
}

Introduction: The aim of this study was to apply low-dose computed tomography (CT) to evaluate treatment and posttreatment effects produced by rapid maxillary expansion (RME) at the levels of the midpalatal suture and the pterygoid processes. Methods: A sample of 17 subjects ( 7 boys, 10 girls; mean age, 11.2 years) was analyzed. Multi-slice CT scans were taken before RME, at the end of the active expansion phase, and after a retention period of 6 months. Statistical analysis was performed with ANOVA for repeated measures with post-hoc tests. Results: The amounts of opening of the midpalatal suture during the active phase of expansion were $3.01,2.17$, and $1.15 \mathrm{~mm}$ for the anterior, middle, and posterior suture widths, respectively. Pterygoid width also showed a statistically significant increase $(1.49 \mathrm{~mm})$. In the postretention period, all transverse measurements had significant decreases except for pterygoid width. Conclusions: At the end of the retention phase after RME therapy, the transverse width of the midpalatal suture was similar to the pretreatment width, whereas the width between the pterygoid processes was significantly increased. (Am J Orthod Dentofacial Orthop 2008;134:389-92)

$\mathbf{R}$ apid maxillary expansion (RME) is the most effective orthopedic procedure to increase the maxillary transverse dimension in young patients by opening the midpalatal suture. ${ }^{1}$ The dentoskeletal effects produced by RME have been investigated primarily by analyzing dental casts or 2-dimensional cephalometric radiographs (posteroanterior or lateral radiographs). ${ }^{2,3}$

Computed tomography (CT) provides a scanning technique of much greater resolution for measurements of transverse dimension in any skeletal structure. ${ }^{4}$ Since 1982, when CT was used for the first time to evaluate the effects of RME, ${ }^{5}$ several studies have described the skeletal and dentoalveolar changes induced by RME in growing and adult patients based on standardized CT scanning registrations. ${ }^{6-11}$ These studies, however, did not look at posttreatment changes, or

\footnotetext{
aPrivate practice, Rome, Italy.

${ }^{\mathrm{b}}$ Assistant professor, Department of Orthodontics, University of Florence, Florence, Italy; Thomas M. Graber Visiting Scholar, Department of Orthodontics and Pediatric Dentistry, School of Dentistry, University of Michigan, Ann Arbor.

'Professor and head, Department of Orthodontics, University of Rome "Tor Vergata," Rome, Italy.

Reprint requests to: Lorenzo Franchi, Università degli Studi di Firenze, Via del Ponte di Mezzo, 46-48, 50127, Firenze, Italy; e-mail, 1.franchi@odonto.unifi.it. Submitted, March 2008; revised and accepted, May 2008. $0889-5406 / \$ 34.00$

Copyright (C) 2008 by the American Association of Orthodontists. doi:10.1016/j.ajodo.2008.05.011
}

had small samples of subjects, and used regular doses of $\mathrm{x}$-ray exposure during the CT examinations.

Our aim in this study was to apply low-dose CT to evaluate the skeletal effects of RME at the levels of the midpalatal suture and the pterygoid processes at the end of the active phase of expansion and after 6 months of retention in 17 growing subjects.

\section{MATERIAL AND METHODS}

A sample of 17 white children ( 7 boys, 10 girls) with a mean age of 11.2 years (range, 8-14 years) who needed orthopedic treatment with RME was selected consecutively at the Department of Orthodontics, "Tor Vergata" Dental School, University of Rome in Italy. The criteria for selection of these patients were constricted maxillary arch, possible unilateral or bilateral posterior crossbite, some crowding, and at least 1 maxillary canine with intraosseous displacement as assessed by panoramic radiographs. The exclusion criteria were age above 15 years, stages in cervical vertebral maturation as assessed on lateral cephalograms more advanced than CS4 (postpubertal), ${ }^{12}$ no maxillary first molars, metallic restorations on the maxillary posterior teeth, previous periodontal disease, previous orthodontic treatment, and craniofacial or growth abnormalities. This project was approved by the Ethical Committee at the University of Rome "Tor 


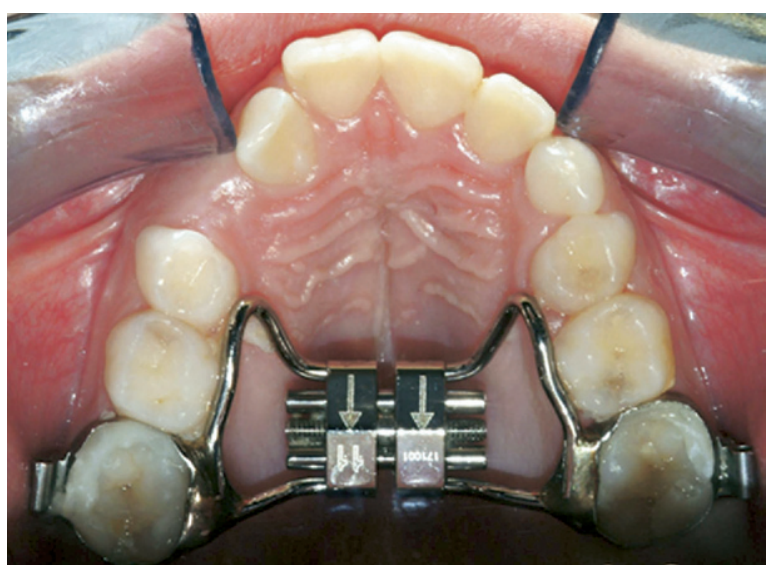

Fig 1. The butterfly rapid maxillary expander.

Vergata," and informed consent was obtained from the parents.

Each patient underwent a standardized protocol with RME performed with the butterfly palatal expander (Fig 1). ${ }^{13}$ The expansion screw was activated 2 turns per day ( $0.25 \mathrm{~mm}$ per turn) for 14 days, for a total expansion of $7 \mathrm{~mm}$ in all subjects. Then the screw was tied off with a ligature wire, and the butterfly expander was kept on the teeth as a passive retainer for 6 months.

Multi-slice CT scans were taken before RME (T0), at the end of the active expansion phase (T1, without removing the expander), and after a retention period of 6 months when the expander was removed (T2). The CT scans were carried out by a trained radiographer at the same scanner console with the primary indication of evaluating the exact position, the 3-dimensional orientation, and the spatial relationships of displaced intraosseous maxillary canines. The low-dose CT scan protocol was described previously. ${ }^{14}$

Measurements were made on the scanned images at skeletal levels, according to definitions in previous studies by Sfondrini et $\mathrm{al}^{6}$ and da Silva Filho et al. ${ }^{9}$

The transverse dimensions of the maxilla were measured on the axial scans parallel to the palatal plane at the midpalatal suture level.

1. Anterior sutural width (ASW): transverse width between the anterior nasal spine points of each maxillary half (Fig 2).

2. Middle sutural width (MSW): transverse width between the points immediately behind the nasopalatine duct (Fig 2).

3. Posterior sutural width (PSW): transverse width between the posterior nasal spine points of each maxillary half (Fig 2).

4. Pterygoid width (PW): transverse width between the lateral pterygoid plates (Fig 3).

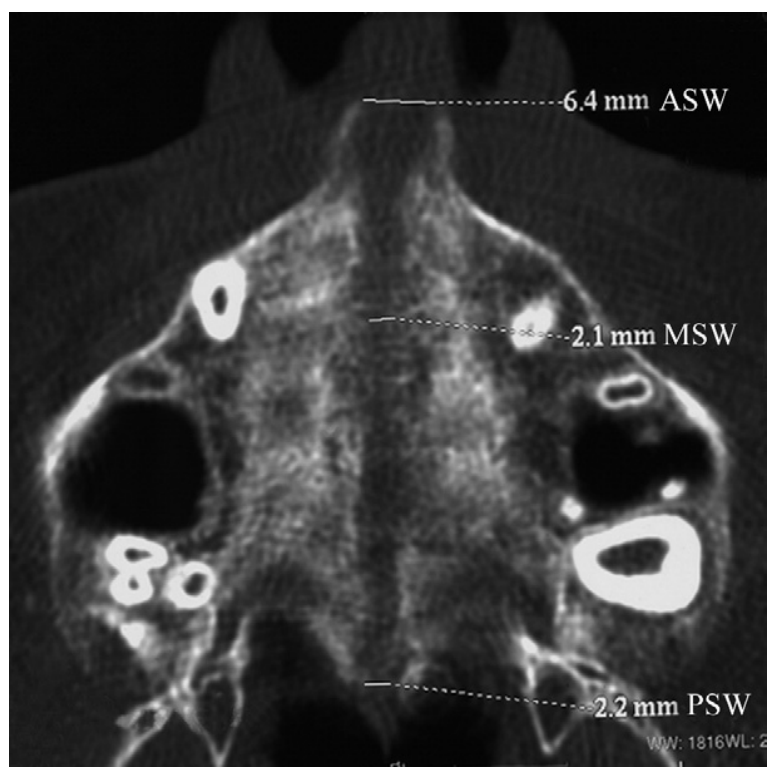

Fig 2. Measurements of the midpalatal suture after RME: anterior suture width (ASW), middle suture width (MSW), and posterior suture width (PSW).

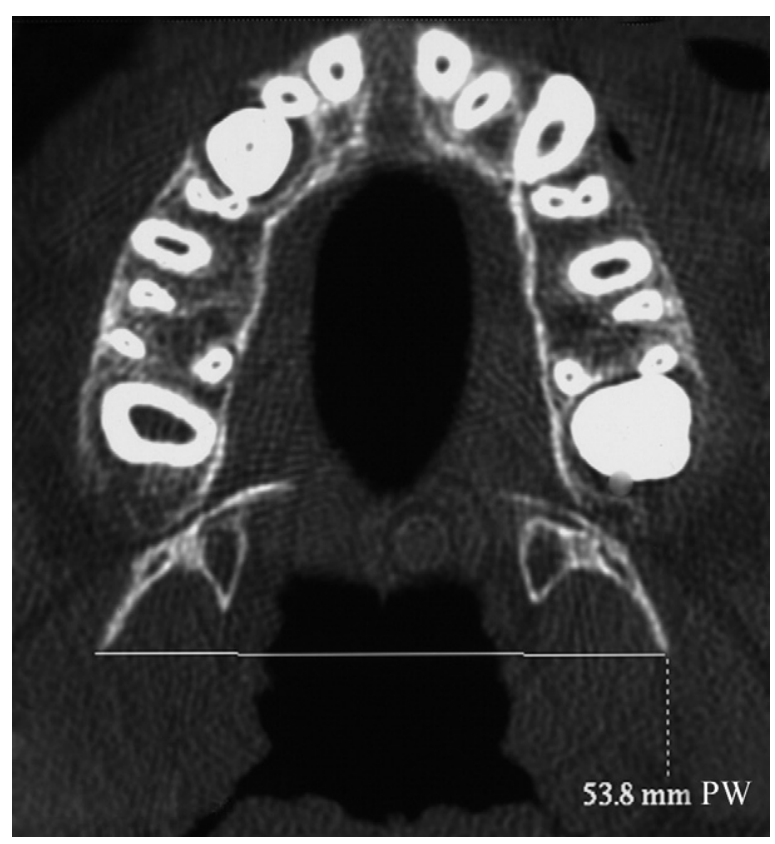

Fig 3. Measurement of pterygoid width (PW).

\section{Statistical analysis}

All measurements were made by 2 operators (F.B. and R.L.) and repeated a month later at the same console by 1 operator (F.B.). Casual and systematic errors were calculated by comparing the first and the 
Table I. Casual and systematic errors calculated comparing the first and the second measurements with paired $t$ test $(\mathrm{t})$ and Dahlberg's formula $(\sigma)$; correlation between the first and the second readings calculated with the Spearman correlation analysis (r)

\begin{tabular}{lrccc}
\hline Variable & $\mathrm{t}$ & Significance & $\sigma$ & $r$ \\
\hline ASW & 1.108 & NS & 0.14 & .932 \\
MSW & 1.033 & NS & 0.16 & .879 \\
PSW & .948 & NS & 0.10 & .943 \\
PW & 1.189 & NS & 0.10 & .805 \\
\hline
\end{tabular}

$N S$, Not significant.

second measurements with paired $t$ tests and Dahlberg's formula. ${ }^{15}$ The correlation between the first and the second readings was calculated by using the Spearman correlation analysis and represented by the $r$ value. All measurement error coefficients were found to be near 1.00 and within acceptable limits (Table I). The mean differences in measurements at T0, T1, and T2 were contrasted with Friedman analysis of variance (ANOVA) for repeated measures followed by the Tukey post-hoc test (SigmaStat 3.5, Systat Software, Point Richmond, Calif). The level of significance was set at $P<0.05$.

\section{RESULTS}

In the evaluation of the changes between $\mathrm{T} 0$ and $\mathrm{T} 1$ (Table II), all linear transverse measurements showed statistically significant increases. The average amounts of opening of the midpalatal suture during the active phase of expansion were 3.01, 2.17, $1.15 \mathrm{~mm}$ for ASW, MSW, and PSW, respectively. PW also showed a statistically significant increase $(1.49 \mathrm{~mm})$.

Between $\mathrm{T} 1$ and $\mathrm{T} 2$, all linear transverse measurements had statistically significant decreases with the exception of PW. At T2, the average values for the widths of the midpalatal suture were not statistically different from those at T0. PW showed statistically significant increments during the overall observation period (T0-T2, $1.12 \mathrm{~mm}$ ).

\section{DISCUSSION}

This study was intended to quantify the skeletal effects of RME and the posttreatment changes after an adequate retention period by using low-dose CT. After 15 days of RME activation ( $7 \mathrm{~mm}$ of expansion at the level of the screw), all linear transverse measurements were significantly increased. The midpalatal suture was opened successfully in all patients with a greater magnitude anteriorly than posteriorly. In agreement with previous authors, the midpalatal suture on the coronal images showed a triangular radiolucent area, where the base is turned forward. ${ }^{6,8-10}$ The amount of expansion at the anterior portion of the suture (ASW, $3.01 \mathrm{~mm}$ ) was variable among the subjects (range, $1.82-4.25 \mathrm{~mm})$. Going from the anterior to the middle to the posterior aspect of the suture, only $43 \%, 31 \%$, and $16 \%$ of the expansion of the screw $(7 \mathrm{~mm})$ could be explained by the distraction across the suture. Most of the expansion, therefore, appeared to be achieved by displacing the dental alveolar complex. The amount of expansion at PSW $(1.15 \mathrm{~mm})$ was about $50 \%$ of that at MSW and about $40 \%$ of that at ASW. The values of sutural expansion during active treatment were similar to those found by Sfondrini et al. ${ }^{6}$ The amount of expansion at the anterior portion of the suture (3.01 $\mathrm{mm}$ ) was greater than the amount reported by Podesser et $\mathrm{al}^{8}(1.52 \mathrm{~mm})$. This difference could be due to the different methods of investigation, since, in the study by Podesser et al, ${ }^{8}$ postexpansion CT examinations were performed after removing the appliance; this might lead to some immediate rebound. da Silva Filho et $\mathrm{al}^{9}$ reported expansion values of $2.21 \mathrm{~mm}$ at $\mathrm{ASW}$ and $0.98 \mathrm{~mm}$ at PSW. These values are smaller than the amount of expansion obtained in our study. All subjects in this study received the same amount of expansion, whereas the amount of expansion in the study of da Silva Filho et $\mathrm{al}^{9}$ was variable.

After the 6-month retention period, the midpalatal suture appeared reorganized with a transverse dimension similar to the pretreatment width; this agreed with the results of da Silva Filho et al. ${ }^{10}$ This confirms previous observations that a retention period of 6 months with the RME in place is necessary to allow a reorganization of the structures involved. ${ }^{16,17}$ The CT examination showed that the maxilla was separated without fractures and bony ridges that could prevent future reexpansion. The favorable sutural changes could be related to the skeletal maturity of these patients who received RME therapy either before or during the pubertal growth spurt. ${ }^{18}$

The distance between the pterygoid processes demonstrated a significant increase at the posttreatment observation with regard to pretreatment values (PW, $1.12 \mathrm{~mm}$ ). The amount of expansion at the pterygoid processes was similar to the amount found by Sfondrini et al. ${ }^{6}$

\section{CONCLUSIONS}

1. Low-dose CT described opening of the midpalatal suture in all subjects after RME therapy. The amount of expansion in the posterior portion of the suture was about $40 \%$ of that observed anteriorly. 
Table II. Statistical comparison of the measurements at T0, T1, and T2 with the Friedman ANOVA for repeated measures followed by the Tukey post-hoc test

\begin{tabular}{|c|c|c|c|c|c|c|c|c|c|}
\hline & \multicolumn{2}{|c|}{ TO } & \multicolumn{2}{|c|}{$T 1$} & \multicolumn{2}{|c|}{$T 2$} & \multicolumn{3}{|c|}{ Comparison of the means } \\
\hline & Mean & $S D$ & Mean & $S D$ & Mean & $S D$ & T0 vs T1 & $T 1$ vs $T 2$ & $T 0$ vs $T 2$ \\
\hline ASW & 0.42 & 0.29 & 3.43 & 0.90 & 0.33 & 0.36 & $*$ & $*$ & NS \\
\hline MSW & 0.19 & 0.18 & 2.36 & 0.59 & 0.21 & 0.31 & $*$ & $*$ & NS \\
\hline PSW & 0.21 & 0.17 & 1.36 & 0.43 & 0.16 & 0.22 & $*$ & $*$ & NS \\
\hline PW & 50.66 & 4.42 & 52.15 & 4.59 & 51.78 & 4.47 & $*$ & NS & $*$ \\
\hline
\end{tabular}

$* P<0.05 ; N S$, not significant.

2. The amount of increase in skeletal widths after RME was limited when compared with the amount of screw activation $(20 \%-50 \%)$ and highly variable among the subjects.

3. After a 6-month retention period, the width of the midpalatal suture was similar to the pretreatment observation, whereas the width at the pterygoid processes was significantly increased.

\section{REFERENCES}

1. Haas AJ. The treatment of maxillary deficiency by opening the midpalatal suture. Angle Orthod 1965;35:200-17.

2. da Silva Filho OG, Montes LA, Torelly LF. Rapid maxillary expansion in the deciduous and mixed dentition evaluated through posteroanterior cephalometric analysis. Am J Orthod Dentofacial Orthop 1995;107:268-75.

3. Sandikcioglu M, Hazar S. Skeletal and dental changes after maxillary expansion in the mixed dentition. Am J Orthod Dentofacial Orthop 1997;111:321-7.

4. Hugo AO, Preston CB, Reis P. A simple and reproducible technique for the use of computed tomography in orthodontics. Eur J Orthod 1981;3:121-4.

5. Timms DJ, Preston CB, Daly PF. A computed tomographic assessment of maxillary movement induced by rapid expansion—a pilot study. Eur J Orthod 1982;4:123-7.

6. Sfondrini G, Schiavi A, Vadalà G. Espansione rapida del palato: controllo degli effetti ortopedici con la TAC. Mondo Ortodontico 1989;14:637-46.

7. Podesser B, Williams S, Bantleon HP, Imof H. Quantitation of transverse maxillary dimensions using computed tomography: a methodological and reproducibility study. Eur J Orthod 2004;26: 209-15.
8. Podesser B, Williams S, Crismani AG, Banteleon HP. Evaluation of the effects of rapid maxillary expansion in growing children using computer tomography scanning: a pilot study. Eur J Orthod 2007;29:37-44.

9. da Silva Filho OG, Lara TL, de Almeida AM, da Silva HC. Evaluation of the midpalatal suture during rapid palatal expansion in children: a CT study. J Clin Pediatr Dent 2005;29:231-8.

10. da Silva Filho OG, Lara TS, da Silva HC, Bertoz FA. Postexpansion evaluation of the midpalatal suture in children submitted to rapid palatal expansion: a CT study. J Clin Pediatr Dent 2006;31:142-8.

11. Garib DG, Henriques JFC, Janson G, Freitas MR, Coelho RA. Rapid maxillary expansion-tooth tissue-borne versus toothborne expanders: a computed tomography evaluation of dentoskeletal effects. Angle Orthod 2005;75:548-57.

12. Baccetti T, Franchi L, McNamara JA. The cervical vertebrae maturation (CVM) method for the assessment of optimal treatment timing in dentofacial orthopedics. Semin Orthod 2005;11: $119-29$.

13. Cozza P, Giancotti A, Petrosino A. Butterfly expander for use in the mixed dentition. J Clin Orthod 1999;33:583-7.

14. Ballanti F, Lione R, Fanucci E, Franchi L, Baccetti T, Cozza P. Immediate and post-retention effects of rapid maxillary expansion investigated by computed tomography in growing patients. Angle Orthod 2008 (in press).

15. Dahlberg G. Statistical methods for medical and biological students. New York: Interscience Publications; 1940.

16. Haas AJ. Long-term post-treatment evaluation of rapid palatal expansion. Angle Orthod 1980;50:189-218.

17. Mew J. Relapse following maxillary expansion. A study of twenty-five consecutive cases. Am J Orthod 1983;83:56-61.

18. Melsen B. Palatal growth studied on human autopsy material. A histologic microradiographic study. Am J Orthod 1975;68:42-54. 